\title{
Solar wind drivers of geomagnetic storms during more than four solar cycles
}

\author{
Ian G. Richardson ${ }^{1,2, *}$, and Hilary V. Cane ${ }^{3}$ \\ 1 Code 661, NASA Goddard Space Flight Center, Greenbelt, MD 20771, USA \\ 2 CRESST and Department of Astronomy, University of Maryland, College Park, MD 20742, USA \\ *corresponding author: e-mail: ian.g.richardson@nasa.gov \\ 3 School of Mathematics and Physics, University of Tasmania, Hobart, Tasmania, Australia
}

Received 24 February 2012 / Accepted 24 April 2012

\begin{abstract}
Using a classification of the near-Earth solar wind into three basic flow types: (1) High-speed streams associated with coronal holes at the Sun; (2) Slow, interstream solar wind; and (3) Transient flows originating with coronal mass ejections (CMEs) at the Sun, including interplanetary CMEs and the associated upstream shocks and post-shock regions, we determine the drivers of geomagnetic storms of various size ranges based on the $K p$ index and the NOAA " $\mathrm{G}$ " criteria since 1964, close to the beginning of the space era, to 2011, encompassing more than four solar cycles (20-23). We also briefly discuss the occurrence of storms since the beginning of the $K p$ index in 1932, in the minimum before cycle 17 . We note that the extended low level of storm activity during the minimum following cycle 23 is without precedent in this 80 -year interval. Furthermore, the "typical" numbers of storm days/cycle quoted in the standard NOAA G storm table appear to be significantly higher than those obtained from our analysis, except for the strongest (G5) storms, suggesting that they should be revised downward.
\end{abstract}

Key words. Solar wind - Interplanetary Coronal Mass Ejection (CME) - Storm - Stream - Solar cycle

\section{Introduction}

In past studies (Richardson 2006; Richardson et al. 2000, 2001, 2002), we divided the near-Earth solar wind since 1972 into three basic flow types in order to assess, for example, the contribution of each type of solar wind flow to long-term $(>\sim$ solar rotation) averages of geomagnetic indices and the interplanetary magnetic field, and to examine the solar wind drivers of geomagnetic storms. The three flow types are: (1) Corotating high-speed streams (typically with solar wind speed $V_{\text {sw }}>\sim 450 \mathrm{~km} \mathrm{~s}^{-1}$ ) (Belcher \& Davis 1971) that originate in coronal holes at the Sun (Krieger et al. 1973; Zirker 1977), and the associated corotating interaction regions; (2) Slower, interstream solar wind, typically associated with the streamer belt at the Sun; and (3) Transient flows originating with coronal mass ejections (CMEs) at the Sun. These include interplanetary coronal mass ejections (ICMEs), the manifestations in the solar wind of CMEs, and the associated upstream shocks and postshock/sheath regions (e.g., Wimmer-Schweingruber et al. 2006; Zurbuchen \& Richardson 2006, and references therein). We collectively term these "CME-associated" flows.

As discussed by Richardson \& Cane (in press) and our earlier studies referenced above, the solar wind flow classification is based on inspection of a variety of data. These include solar wind plasma and magnetic field data from the OMNI2 database (http://omniweb.gsfc.nasa.gov/; King \& Papitashvili 2005), geomagnetic activity indices, and energetic particle observations from neutron monitors and spacecraft which can indicate the passage of high-speed streams and CME-associated flows past the Earth. Richardson \& Cane (in press) also discuss how the classification has recently been extended back to the beginning of the OMNI2 data, in November 1963, as well as being updated to near present, producing a classification of solar wind flows extending over more than four solar cycles from the minimum before cycle 20 to the ascending phase of cycle 24 .

In this paper, we use this solar wind classification to identify the types of flows driving geomagnetic storms of various ranges of sizes during the period 1964-2011, updating the results of Richardson et al. (2002) who considered the period from 1972 to 2000 . We also extend the storm analysis back to the beginning of the $K p$ index in 1932 and discuss storm rates over an 80-year interval encompassing cycles 17-23.

\section{Solar wind drivers of geomagnetic storms in 1963-2011}

As in Richardson et al. (2002), we identify geomagnetic storms using the 3-h $K p$ index (Bartels et al. 1939; Menvielle \& Berthelier 1991; Rostoker 1972). We employ two methods of defining storm strength:

- The criteria of Gosling et al. (1991): A "major" storm is defined by $K p_{\max } \geq 8$ and $K p \geq 6$ for at least three 3 -h intervals in a $24-\mathrm{h}$ period. A "large" storm has $7 \leq K p_{\max } \leq 7+$, and $K p \geq 6$ for at least three $3-\mathrm{h}$ intervals in a 24-h period. "Medium" storms are all other cases with $K p_{\max } \geq 6-$. A "small" storm has $5-\leq K p_{\max } \leq 5+$. Note that these criteria identify days when storm conditions prevail ("storm days") rather than discrete storms characterized by a rise then fall in activity and some maximum activity level. Thus, a storm extending over several days may contribute to more than one day of storm conditions. 




Fig. 1. Occurrence rates (/year) of small $(\sim \mathrm{G} 1)$, medium $(\sim \mathrm{G} 2)$, and large + major $(\geq \mathrm{G} 3)$ geomagnetic storms (storm days) in 1964-2011 associated with CME-associated flows and corotating streams. The monthly sunspot number is on the top panel. (Updated from Richardson et al. 2001; Richardson 2006.)

- The NOAA “G” storm sizes (http://www.swpc.noaa.gov/ NOAAscales/). Specifically, G5 ("extreme") has $K p=9$; G4 ("severe") has $K p=8$ including a 9-; G3 ("strong") reaches $K p=7 ; \mathrm{G} 2$ ("moderate") has $K p=6$; and G1 ("minor") has $K p=5$. Again we consider storm days based on these criteria. Note that G4 and G5 storms are comparable to a "major" storm in the Gosling et al. (1991) criteria, G3 is comparable to a large storm, G2 to a medium storm, and G1 to a small storm.

To identify the solar wind drivers of these geomagnetic storms, we used an automated process which identifies storms as defined above in the $K p$ index, and then compares the storm time against the solar wind flow classification to identify the driver type. If more than one flow type is present, that associated with the highest activity levels is chosen. For discussion of the interplanetary causes of geomagnetic storms, see, for example, Tsurutani \& Gonzalez (1997); Zhang et al. (2007); Echer et al. (2008), and references therein.

Figure 1 shows the annual occurrence rate of storms (storm days) of different Gosling et al. (1991) sizes driven by CMEassociated flows or corotating streams from 1964 to 2011 together with the monthly sunspot number in the top panel. As noted above, "large" and "major storms", which are summed together in Figure 1 because of limited statistics, approximately correspond to $\geq \mathrm{G} 3$ storms, while medium and small storms are approximately $\mathrm{G} 2$ and $\mathrm{G} 1$, respectively.
We do not show storms associated with slow solar wind in Figure 1 because, as will be discussed below, relatively few storms are generated by slow solar wind.

Generally, the number of CME-associated storms (black curves in Fig. 1) follows solar activity levels, as would be expected since the ICME rate at 1 AU (Richardson \& Cane 2010) and the CME rate at the Sun (Robbrecht et al. 2009; Webb \& Howard 1994; Yashiro et al. 2004) increase from solar minimum to solar maximum. Furthermore, Figure 1 indicates that the maximum rate of storms driven by CMEassociated flows approximately follows the size of the sunspot cycle, i.e. storm rates are higher in cycles 21 and 22 than in cycles 20 and 23 . Both cycles 21 and 22, and possibly cycle 20 , show evidence of a brief decrease in $\geq$ medium storm activity near solar maximum. Such a feature is less evident in cycle 23. As has been noted previously (e.g., Richardson et al. 2000, 2002), this feature may be related to what has been referred to as the "Gnevyshev Gap" (Feminella \& Storini 1997), characterized by a lack of energetic solar phenomena near solar maximum, that may be associated with the decrease in several solar indices near solar maximum discussed by Gnevyshev (1967, 1977). Richardson et al. (2002) (see also Richardson \& Cane in press) suggested that this decrease in the level of geomagnetic activity near solar maximum is related to a temporary decrease in solar and interplanetary magnetic fields and solar wind speed, including in CME-associated flows. 


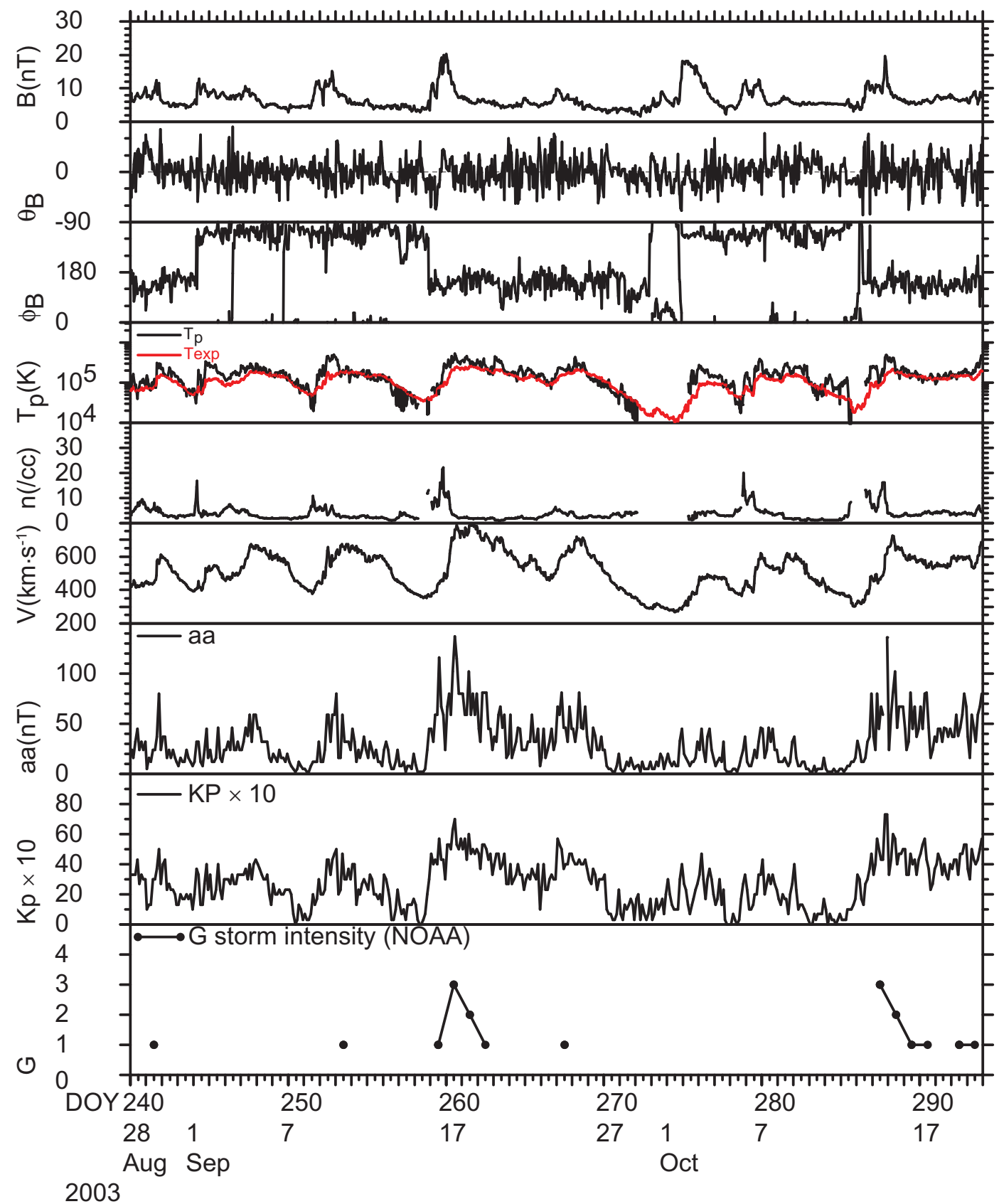

Fig. 2. Solar wind and geomagnetic $(a a, K p \times 10)$ indices for a two solar rotation interval in August-October 2003 dominated by a sequence of corotating high-speed streams with peak speeds $\sim 600-800 \mathrm{~km} \mathrm{~s}^{-1}$. Geomagnetic activity frequently reached G1-G3 levels (bottom panel). The solar wind parameters shown are: the magnetic field intensity, polar and azimuthal angles (GSE coordinates), proton temperature with expected temperature (Richardson \& Cane 1995) in red; density and solar wind speed.

Stream-associated storms (red curves in Fig. 1) are typically most prominent for 3-4 years during the declining phase of the cycle, but unusually were only prominent for one year (2003) in cycle 23 (Kozyra et al. 2006). Figure 2 shows solar wind and geomagnetic data (aa, Mayaud 1972 and $K p \times 10$ indices) for a representative two solar rotation period in August-October, 2003 dominated by a sequence of corotating high-speed streams (panel 6) with peak speeds of $\sim 600-800 \mathrm{~km} \mathrm{~s}^{-1}$. The enhanced geomagnetic activity during passage of these streams reached G1 levels on nine days, and G2 and G3 each on two days, as indicated in the bottom panel. Geomagnetic activity associated with individual streams in 2003 has also been discussed by Richardson et al. (2006) and Tsurutani et al. (2011a).
Returning to Figure 1, small storms produced by streams predominate over those arising from CME-associated flows, whereas CME-associated flows become increasingly more dominant for larger storms.

Cycle 20 has a smaller occurrence of CME-associated storms than the other cycles, but there were prominent stream-associated storms during the declining phase. On the other hand, the other weaker cycle, 23, has just a brief stream-associated storm peak in the declining phase, as noted above, whereas CME-associated storms continued to be observed past the stream-associated peak and into the late declining phase of the cycle. Thus, overall, each cycle shows variations in the relative contributions from CME- and 




Fig. 3. Solar wind and geomagnetic $(a a, K p \times 10)$ indices for a two solar rotation interval during May-June in 2009 , the year of minimum storm activity since at least 1964, in the same format as Figure 2 . Note the absence of high-speed streams, although weak recurrent streams that rarely exceed $400 \mathrm{~km} \mathrm{~s}^{-1}$ can be identified. One G1 storm occurred during this period, associated with the interaction region ahead of a corotating stream.

stream-associated storms. The contribution of different types of flow to various size storms around solar maximum and minimum will be discussed further below. In this analysis, 2009 had the lowest annual number of small storms (2) (and also G1 storms (3)) since the beginning of the space era while the largest number of large + major storms (31) occurred in 1991. Figure 3 shows solar wind and geomagnetic data for a two rotation interval in 2009, illustrating the lack of highspeed streams, in contrast to Figure 2, though corotating structures including weak streams that rarely exceed $400 \mathrm{~km} \mathrm{~s}^{-1}$ can still be identified in the plasma and magnetic field data. As also noted by Tsurutani et al. (2011b), the low geomagnetic activity levels in 2009 can be attributed to the predominance of slow solar wind at the expense of streams, and the unusually weak interplanetary magnetic field strength.

As noted above, the solar cycle variation in the number of geomagnetic storms in cycles such as 21 and 22 may include two peaks due to CME-associated storms, separated by the Gnevyshev gap, and a separate peak during the declining phase of the cycle associated with corotating streams (cf. Fig. 1). This peak, however, may overlap in time with the second CMEassociated storm peak, producing the "dual peak" distribution of storms during the solar cycle noted, for example, by Gonzalez et al. (1990); variations in the total number of storms are illustrated below in Figure 5. It is evidently incorrect simply to ascribe the first peak to CME-associated storms and the 
I.G. Richardson and H.V. Cane: Geomagnetic storms over four solar cycles

\section{Solar Minimum Storms}

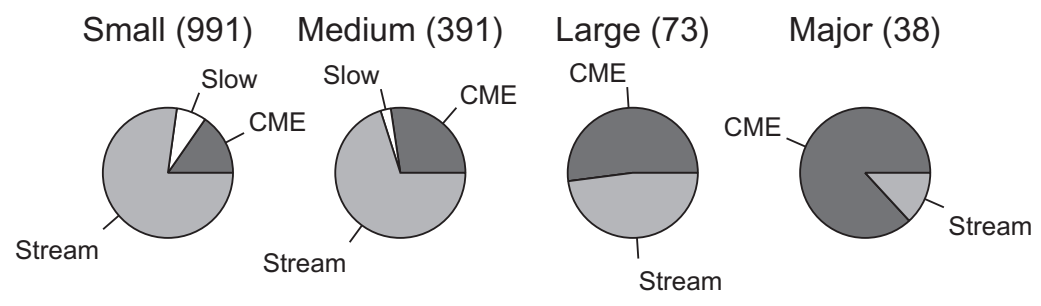

\section{Solar Maximum Storms}

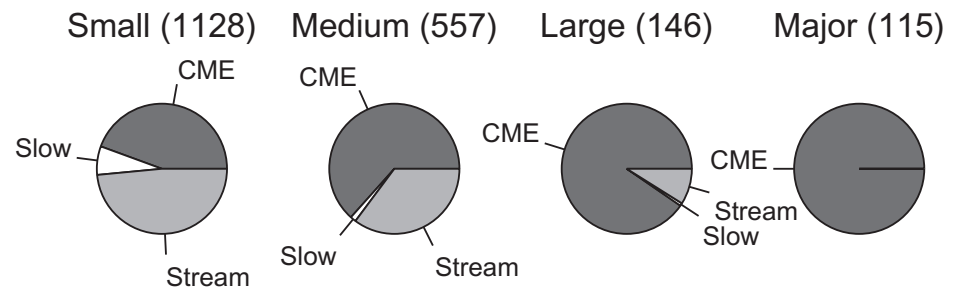

\section{Solar Minimum Storms}

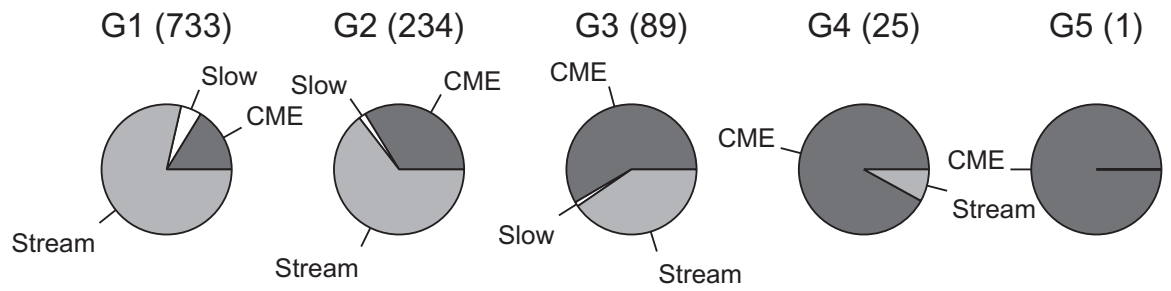

\section{Solar Maximum Storms}

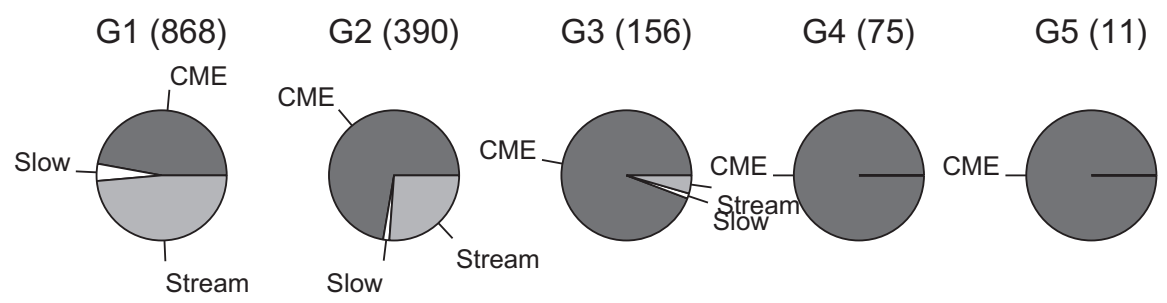

Fig. 4. Pie plots summarizing the solar wind drivers of geomagnetic storms (sized by the Gosling et al. (1991) criteria (top two rows) or the NOAA G scale (bottom two rows)) around solar minimum (top row of each pair) and solar maximum (bottom row of each pair) during 19642011. Numbers in brackets indicate the number of storms included in each pie plot. Dark gray = CME-associated flows; light gray = streamassociated; white = slow solar wind. "Unclear" events have been removed.

second to stream-associated storms. We also note that Echer et al. (2011) have discussed variations in the solar cycle occurrence of storms of various strengths based on the $D_{\text {st }}$ index during 1957-2008. They conclude that the more intense storms tend to follow the solar cycle, whereas less intense storms are most prevalent during the declining phase, consistent with the results shown in Figure 1 based on $K p$.

Figure 4 and Table 1 summarize the solar wind structures associated with storms of different Gosling et al. (1991) (upper rows of figure and table) or NOAA G sizes (lower rows), during solar minimum or solar maximum intervals in 1964-2011, with "unclear" events removed. The solar minimum intervals are (arbitrarily) bounded by the years in which the smoothed sunspot number fell below or rose above 40 (cf. Fig. 1), i.e., 1962 (though the analysis commenced in 1964)-1966, 1973-1977, 1984-1987, 1993-1997, and 2004-2010. Intervening periods are the "solar maximum" intervals. Numbers in brackets in Figure 4 show the number of storms included in each pie plot, and indicate the increasing prevalence of larger storms around solar maximum. At solar minimum, streams are responsible for around three-quarters of small $(\sim 77 \%)$ or medium $(\sim 70 \%)$ storms, around a half ( $48 \%$ ) of large storms, and $\sim 13 \%$ of major storms, the remainder being predominantly associated with CME flows. At solar maximum, streams are still responsible for around half $(48 \%)$ of small storms, but for around a third $(35 \%)$ of medium storms and only $9 \%$ of large or major storms. Considering the $\mathrm{G}$ storm levels, at solar minimum, over threequarters (79\%) of G1 storms are stream-associated, as are around two-thirds (65\%) of G2 and $40 \%$ of G3 storms. G4 and G5 storms are nearly exclusively (96\%) driven by CME-associated flows. At solar maximum, around a half (49\%) of G1 storms are stream-associated, as are around a quarter (26\%) of G2 storms. Otherwise, these storms are driven by CME-associated flows, as are the vast majority (96\%) of C3-C5 storms. Thus, these results again show the different contribution of streams and CME-associated flows at solar minimum and maximum, though 
Table 1. Association of geomagnetic storms and solar wind flows in 1964-2011.

\begin{tabular}{|c|c|c|c|c|c|c|}
\hline & Storm size & Events & CME-associated (\%) & Stream (\%) & Slow S.W. (\%) & Uncertain events \\
\hline \multirow[t]{4}{*}{ Solar minimum } & Small & $991 *$ & 15 & 77 & 8 & 91 \\
\hline & Medium & 391 & 27 & 70 & 3 & 39 \\
\hline & Large & 73 & 52 & 48 & 0 & 3 \\
\hline & Major & 38 & 87 & 13 & 0 & 3 \\
\hline \multirow[t]{4}{*}{ Solar maximum } & Small & 1128 & 44 & 48 & 7 & 110 \\
\hline & Medium & 557 & 63 & 35 & 1 & 33 \\
\hline & Large & 146 & 90 & 9 & 1 & 4 \\
\hline & Major & 115 & 100 & 0 & 0 & 0 \\
\hline \multirow[t]{5}{*}{ Solar minimum } & G1 & 733 & 16 & 79 & 5 & 64 \\
\hline & G2 & 234 & 34 & 65 & 2 & 25 \\
\hline & G3 & 89 & 58 & 40 & 1 & 6 \\
\hline & G4 & 25 & 92 & 8 & 0 & 0 \\
\hline & G5 & 1 & 100 & 0 & 0 & 0 \\
\hline \multirow[t]{5}{*}{ Solar maximum } & G1 & 868 & 47 & 49 & 4 & 72 \\
\hline & G2 & 390 & 72 & 26 & 2 & 26 \\
\hline & G3 & 156 & 94 & 5 & 1 & 3 \\
\hline & G4 & 75 & 100 & 0 & 0 & 0 \\
\hline & G5 & 11 & 100 & 0 & 0 & 0 \\
\hline
\end{tabular}

*Excluding "uncertain" events.

CME-associated flows tend to be responsible for the most severe storms throughout the solar cycle. This conclusion is consistent with other studies, such as that of Zhang et al. (2007) which found that only $\sim 13 \%$ of intense $\left(D_{\text {st }} \leq-100 \mathrm{nT}\right)$ geomagnetic storms in 1996-2005 were driven by streams, while the remainder involved CME-associated flows (ICMEs and/or upstream sheaths) (see also Echer et al. 2008). We also note that occasionally (e.g., two of the 88 Zhang et al. 2007 events) both CME-associated flows and streams may be involved in the production of a storm, a complexity discussed, for example, by Burlaga et al. (1987); Dal Lago et al. (2006); and Richardson (2006), but not explicitly considered in our analysis where the structure associated with the more intense activity in $K p$ is identified as the driver of the storm.

\section{Geomagnetic storms since 1932}

Since the $K p$ index goes back to 1932, we can also examine the occurrence of storms of different sizes back to the minimum before solar cycle 17. Figure 5 shows the annual number of storms of different Gosling et al. (1991) (top panel) and NOAA $\mathrm{G}$ sizes (third panel) since the beginning of the $K p$ index in 1932, including solar cycles from 17 (the second panel shows the yearly sunspot number; vertical lines indicate the year of maximum sunspot number). We cannot identify the storm drivers directly before 1964 because of the lack of solar wind data. However, based on the results in Figure 1, the stronger Gosling et al. (1991) storms are likely to be predominantly related to CME-associated flows throughout the solar cycle, while small storms may be predominantly related to CME flows at active times and to streams during the declining phase of the solar cycle. Dual peaks were also clearly observed in the rate of the largest (likely CME-associated) storms around the maxima of cycles 18 and 19. Figure 5 suggests that the persistent interval of low activity during the recent solar minimum is unprecedented during this 80 -year period. In particular, the years from 2008 to 2011 had the smallest annual numbers of "small" storms (12, 2, 10, and 21, respectively) based on $K p$ according to this analysis, whereas the previous record low number of small storms (22) was in 1966. This indicates that the solar wind conditions and geomagnetic activity in the recent minimum were not only the most unusual since the beginning of the space era but also from at least 1932. The $\mathrm{G}$ storm rates show generally similar features, including the unusually low levels of storm activity in the recent minimum. Interestingly, the G1 storm rate in 2003, the single year dominated by stream associated flows during the declining phase of cycle 23 (Fig. 1), was the highest since at least 1932 .

The bottom panel of Figure 5 shows the number of storms of different $G$ sizes in each solar cycle (defined to start at the year of minimum yearly sunspot number). Though fewer storms occurred in the weaker cycles 17,20 , and 23 , at least for $\leq \mathrm{G} 4$ storms, overall, the number of storms/cycle does not strictly follow cycle-to-cycle variations in the size of the sunspot cycle. In particular, the number of the most geoeffective (G5) storms has little correlation $(c c=0.299)$ with the size of the related sunspot cycle, though the number of events is small. Thus, advance prediction of the size of a solar cycle is likely to be only a weak indicator of the likely number of the especially important, most intense geomagnetic storms that might be expected to occur during the cycle.

An interesting aspect of the numbers of storms/cycle in the bottom panel of Figure 5 is that they suggest that the "typical" numbers of storm days during each solar cycle for the different $\mathrm{G}$ storm sizes quoted in the NOAA storm table (http:// www.swpc.noaa.gov/NOAAscales/) are significantly overestimated. The first column of Table 2 shows the number of days/cycle that each storm level (quote) "is met" given in the NOAA storm table. The average numbers of days/cycle for which activity was at (and did not exceed) each $\mathrm{G}$ storm level in cycles 17-23 are given in the second column. Evidently our rates in column 2 are $~ 50 \%$ of those given in the NOAA storm table except for G5 storms. (Selecting say only the space era cycles 20-23 does not change this conclusion, cf. Fig. 5.) However, "meeting" the storm level in the NOAA table is also ambiguous since this could also include days in which a 
I.G. Richardson and H.V. Cane: Geomagnetic storms over four solar cycles
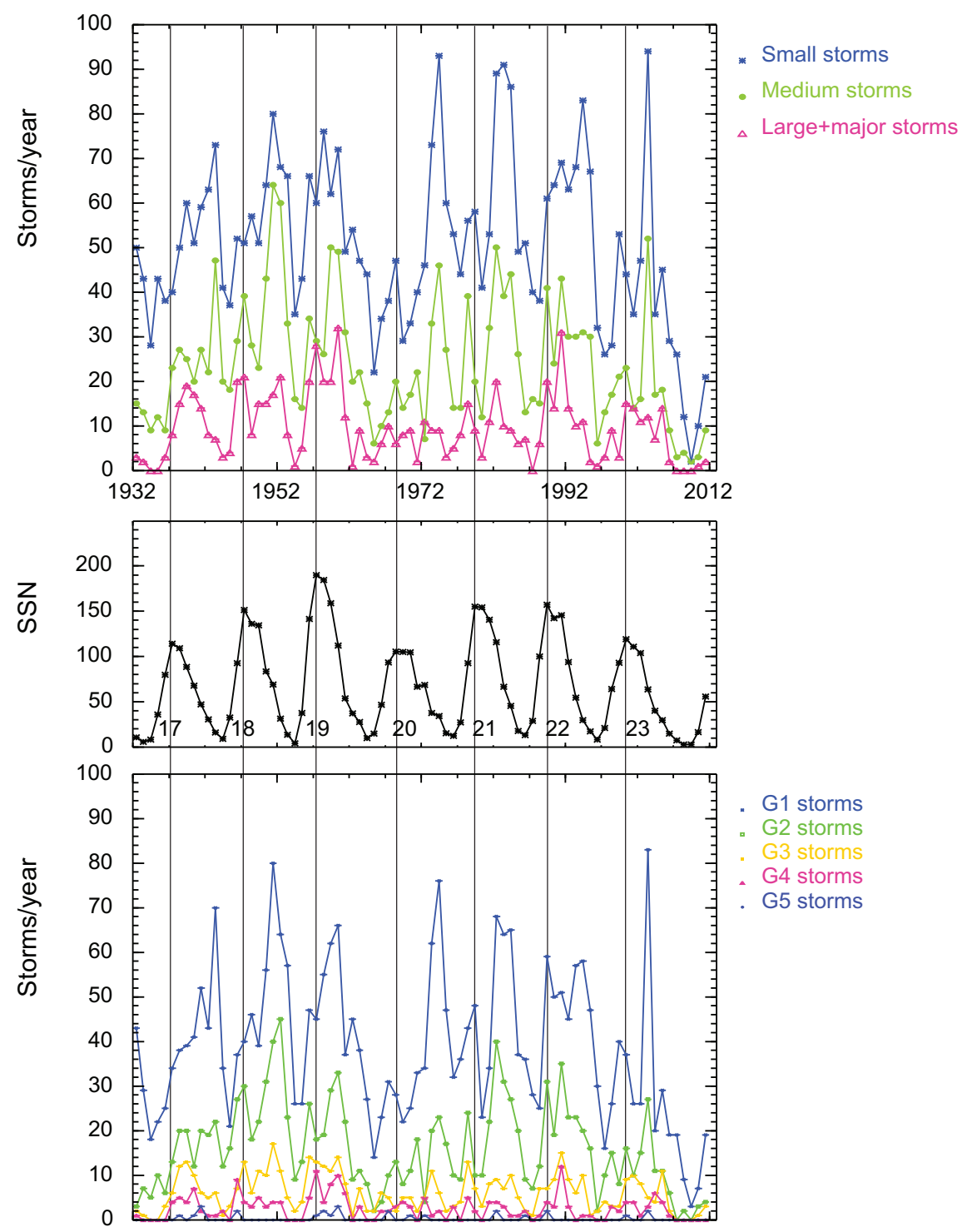

G1 storms

G2 storms

3 storms

G4 storms

- G5 storms

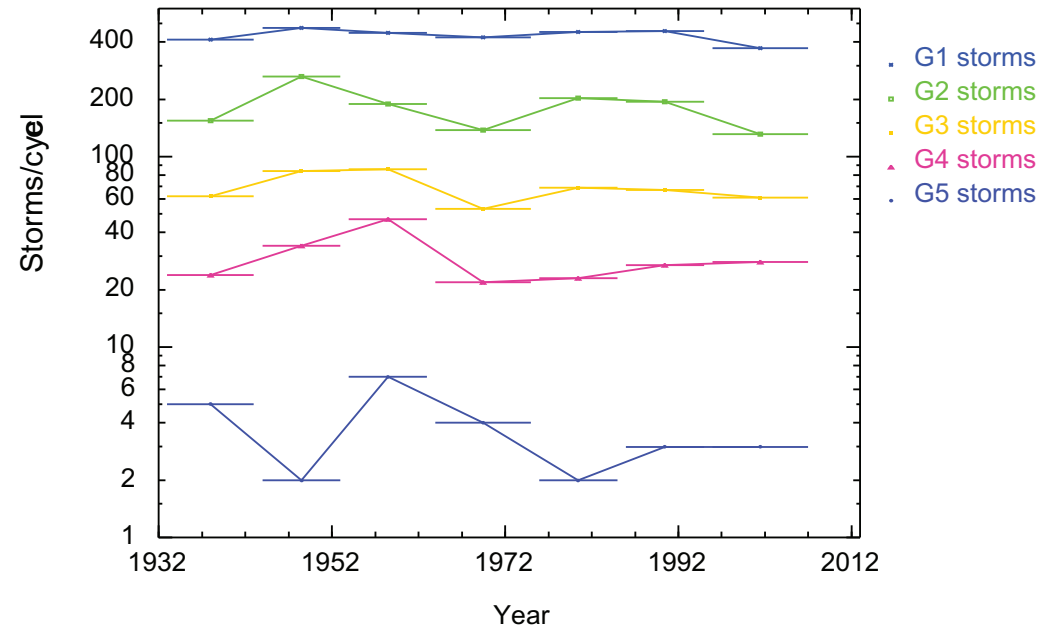

Fig. 5. Annual numbers of storms (storm days) of different Gosling et al. (1991) sizes from 1932, the beginning of the $K p$ index, to 2011 (top panel) and similarly for NOAA G storm sizes (third panel). The second panel shows the yearly sunspot number (vertical lines indicate years of sunspot maximum), while the bottom panel shows the number of $\mathrm{G}$ storms/solar cycle. Note that the persistent low levels of storm activity during the recent solar minimum are unprecedented during this 80-year period. In particular, 2008-2011 had the lowest number of small or G1 storms recorded in the $K p$ index based on this analysis. 
Table 2. Number of G storm days/cycle.

\begin{tabular}{lccc}
\hline \hline $\begin{array}{l}\text { NOAA } \\
\text { storm } \\
\text { size G }\end{array}$ & $\begin{array}{c}\text { NOAA table } \\
\text { days/cycle }\end{array}$ & $\begin{array}{c}\text { Size }=\mathrm{G} \\
(1933-2007) \\
\text { days/cycle }\end{array}$ & $\begin{array}{c}\text { Size } \geq \mathrm{G} \\
(1933-2007) \\
\text { days/cycle }\end{array}$ \\
\hline 1 & 900 & $433 \pm 32$ & $717 \pm 55$ \\
2 & 360 & $182 \pm 43$ & $284 \pm 45$ \\
3 & 130 & $69 \pm 11$ & $102 \pm 14$ \\
4 & 60 & $29 \pm 8$ & $33 \pm 8$ \\
5 & 4 & $3.7 \pm 1.7$ & $3.7 \pm 1.7$ \\
\hline
\end{tabular}

particular storm level is exceeded. Thus, the third column of Table 2 shows the number of days when each storm level was equaled or exceeded. However, the NOAA rates still exceed these by $\sim 25 \%$ to $80 \%$, except for G5 storms. Thus, we suggest that the numbers of storm days/cycle quoted in the NOAA table should be revised downward.

\section{Summary}

- The solar wind structures driving geomagnetic storms (based on the $K p$ index) over four solar cycles have been identified, and the varying importance of CME-related flows, high-speed streams, and slow solar wind in driving storms of different strengths during the solar cycle has been investigated.

- Storms driven by CME-associated flows have an occurrence rate that generally follows the solar activity cycle but may be temporarily depressed for a period around solar maximum. As the storm size increases, CME-associated flows contribute to a larger fraction of events.

- Storms driven by corotating high-speed streams typically predominate for 3-4 years during the declining phase of the cycle and are the predominant drivers of weaker storms.

- The declining phase of cycle 23 and following minimum is characterized by: an unusually brief ( $\sim 1$ year) interval (in 2003) dominated by stream-driven storms (2003 had the highest number of G1 storms since at least 1932); an extended occurrence of CME-associated storms; and the lowest annual number of storms, in 2009, not only since the beginning of the space era but also since the beginning of the $K p$ index in 1932. The absence of high-speed corotating streams contributes to the low geomagnetic activity levels in 2009.

- The average number of storm days of different NOAA G sizes/solar cycle inferred from observations in 19332007 (cycles 17-23) is around half of that stated on the NOAA storm scale table, considering storms with a particular $\mathrm{G}$ storm level. If days that equal or exceed a given storm level are considered, the NOAA table still overestimates the number of storm days for each $\mathrm{G}$ level by $\sim 25 \%$ to $80 \%$, except for G5 storms. The reason for this is unclear.

- The number of storm days/cycle is only weakly correlated with the peak sunspot number and in particular does not faithfully follow cycle-to-cycle variations in the sunspot number. The small number of the G5 storms/cycle is not significantly correlated with cycle size. Hence, the number of especially intense geomagnetic/space weather events in a new cycle cannot be reliably estimated from a prediction of the cycle size.
Acknowledgements. We thank the many researchers who have made available the near-Earth magnetic field, plasma and energetic particle data that have contributed to the solar wind identifications. The geomagnetic data were obtained from the National Geophysical Data Center (http://ngdc.noaa.gov) and the International Service for Geomagnetic Indices (http://isgi.cetp.ipsl.fr/). The solar wind data were obtained from the OMNI2 database at the Space Physics Data Facility, Goddard Space Flight Center (http://omniweb.gsfc.nasa.gov/).

\section{References}

Bartels, J., N.H., Heck, and H.F., Johnston, The three-hour range index measuring geomagnetic activity, J. Geophys. Res., 44, 411, 1939.

Belcher, J.W., and L. Davis, Large amplitude Alfvén waves in the interplanetary medium, 2, J. Geophys. Res., 76, 3534, 1971.

Burlaga, L.F., K.W. Behannon, and L.W. Klein, Compound streams, magnetic clouds, and major magnetic storms, J. Geophys. Res., 92, 5725, 1987.

Dal Lago, A., W.D. Gonzalez, L.A. Balmaceda, L.E.A. Vieira, E. Echer, F.L. Guarnieri, et al., The 17-22 October (1999) solarinterplanetary-geomagnetic event: Very intense geomagnetic storm associated with a pressure balance between interplanetary coronal mass ejection and a high-speed stream, J. Geophys. Res., 111, A07S14, DOI: 10.1029/2005JA011394, 2006.

Echer, E., W.D. Gonzalez, B.T. Tsurutani, and A.L.C. Gonzalez, Interplanetary conditions causing intense geomagnetic storms $\left(D_{\mathrm{st}} \leq-100 \mathrm{nT}\right)$ during solar cycle 23 (1996-2006), J. Geophys. Res., 113, A05221, DOI: 10.1029/2007JA012744, 2008.

Echer, E., W.D. Gonzalez, and B.T. Tsurutani, Statistical studies of geomagnetic storms with peak $D_{\mathrm{st}} \leq-50 \mathrm{nT}$ from 1957 to 2008 , J. Atmos. Sol.Terr. Phys., 73, 1454, 2011.

Feminella, F., and M. Storini, Large scale dynamical phenomena during solar activity cycles, Astron. Astrophys., 322, 311, 1997.

Gnevyshev, M.N., On the 11-years cycle of solar activity, Sol. Phys., 1, 107, 1967.

Gnevyshev, M.N., Essential features of the 11 year solar cycle, Sol. Phys., 51, 175, 1977.

Gonzalez, W.D., A.L.C. Gonzalez, and B.T. Tsurutani, Dual peak solar cycle distribution of intense geomagnetic storms, Planet. Space Sci., 38, 181, 1990.

Gosling, J.T., D.J. McComas, J.L. Phillips, and S.J. Bame, Geomagnetic activity associated with Earth passage of interplanetary shock disturbances and coronal mass ejections, J. Geophys. Res., 96, 7831, 1991.

King, J.H., and N.E. Papitashvili, Solar wind spatial scales in and comparisons of hourly Wind and ACE plasma and field data, J. Geophys. Res., 110, 2104, 2005.

Kozyra, J.U., G. Crowley, B.A. Emery, X. Fang, G. Maris, et al., Response of the upper/middle atmosphere to coronal holes and powerful high-speed solar wind streams in 2003, in Recurrent Magnetic Storms: Corotating Solar Wind Streams, ed. B.T. Tsurutani, R.L. McPherron, W.D. Gonzalez, G. Lu, J.H.A. Sobral, and N. Gopalswamy, A.G.U. Geophysical Monograph, 167, 319, 2006.

Krieger, A.S., A.F. Timothy, and E.C. Roelof, A coronal hole and its identification as the source of a high velocity solar wind stream, Sol. Phys., 29, 505, 1973.

Mayaud, P.N., The $a a$ indices: A 100-year series characterising the geomagnetic activity, J. Geophys. Res., 77, 6870, 1972.

Menvielle, M., and A. Berthelier, The K-derived planetary indices: Description and availability, Rev. Geophys., 29, 415, DOI: 10.1029/91RG00994, 1991.

Richardson, I.G., The formation of CIRs at stream-stream interfaces and resultant geomagnetic activity, in Recurrent Magnetic Storms: Corotating Solar Wind Streams, ed. B.T. Tsurutani, R.L. McPherron, W.D. Gonzalez, G. Lu, J.H.A. Sobral, and N. Gopalswamy A.G.U. Geophysical Monograph, 167, 45, 2006. 
I.G. Richardson and H.V. Cane: Geomagnetic storms over four solar cycles

Richardson, I.G., and H.V. Cane, Near-Earth interplanetary coronal mass ejections during solar cycle 23 (1996-2009): Catalog and summary of properties, Sol. Phys., 264, 189, 2010.

Richardson, I.G., and H.V. Cane, Near-Earth solar wind flows and related geomagnetic activity over more than four solar cycles (1964-2011), J. Space Weather Space Clim., in press.

Richardson, I.G., E.W. Cliver, and H.V. Cane, Sources of geomagnetic activity over the solar cycle: Relative importance of CMEs, high-speed streams, and slow solar wind, J. Geophys. Res., 105, 18203, 2000.

Richardson, I.G., E.W. Cliver, and H.V. Cane, Sources of geomagnetic storms for solar minimum and maximum conditions during 1972-2000, Geophys. Res. Lett., 28, 2569, 2001.

Richardson, I.G., H.V. Cane, and E.W. Cliver, Sources of geomagnetic activity during nearly three solar cycles (1972-2000), J. Geophys. Res., 107, 1187, DOI: 10.1029/2001JA000504, 2002.

Richardson, I.G., D.F. Webb, J. Zhang, D.B. Berdichevsky, D.A. Biesecker, J.C. Kasper, et al., Major geomagnetic storms $\left(D_{\mathrm{st}} \leq\right.$ $-100 \mathrm{nT}$ ) generated by corotating interaction regions, J. Geophys. Res., 111, A07S09, DOI: 10.1029/2005JA011476, 2006.

Robbrecht, E., D. Berghmans, and R.A.M. Van der Linden, Automated LASCO CME catalog for solar cycle 23: Are CMEs scale invariant? Astrophys. J., 691, 1222-1234, DOI: 10.1088/0004-637X/691/2/1222, 2009.

Rostoker, G., Geomagnetic indices, Rev. Geophys., 10, 935, DOI: 10.1029/RG010i004p00935, 1972.

Tsurutani, B.T., and W.D. Gonzalez, The interplanetary causes of magnetic storms: A review, In Magnetic Storms, ed. B.T. Tsurutani, W.D. Gonzalez, Y. Kamide, and J.K. Arballo, A.G.U. Geophys. Monogr. Ser., Vol. 98, Washington, D.C., AGU, 77, 1997.
Tsurutani, B.T., E. Echer, F.G. Guarnieri, and W.D. Gonzalez, The properties of two solar wind high speed streams and related geomagnetic activity during the declining phase of solar cycle 23 , J. Atmos. Sol. Terr. Phys., 73, 164, DOI: 10.1016/j.jastp.2010.04.003, 2011a.

Tsurutani, B.T., E. Echer, and W.D. Gonzalez, The solar and interplanetary causes of the recent minimum in geomagnetic activity (MGA23): a combination of midlatitude small coronal holes, low IMF $B_{z}$ variances, low solar wind speeds and low solar magnetic fields, Ann. Geophys., 29, 839, DOI: 10.5194/angeo29-839-2011, 2011b.

Webb, D.F., and R.A. Howard, The solar cycle variation of coronal mass ejections and the solar wind mass flux, J. Geophys. Res., 99, 4201, 1994

Wimmer-Schweingruber, R.F., N.U. Crooker, A. Balogh, V. Bothmer, R.J. Forsyth, et al., Understanding interplanetary coronal mass ejection signatures, Space Sci. Rev., 123, 177-216, 2006.

Yashiro, S., N. Gopalswamy, G. Michalek, O.C. St. Cyr, S.-P. Plunkett, et al., A catalog of white light coronal mass ejections observed by the SOHO spacecraft, J. Geophys. Res., 109, A07105, DOI: 10.1029/2003JA010282, 2004.

Zhang, J., I.G. Richardson, D.F. Webb, N. Gopalswamy, E. Huttunen, et al., Solar and interplanetary sources of major geomagnetic storms $\left(D_{\mathrm{st}} \leq-100 \mathrm{nT}\right)$ during 1996--2005, J. Geophys. Res., 112, A12105, DOI: 10.1029/2007JA012332, 2007.

J.B., Zirker, (ed.), Coronal Holes and High Speed Wind Streams, Skylab Solar Workshop, Colorado University Press, Boulder, CO, 1977.

Zurbuchen, T.H., and I.G. Richardson, In-situ solar wind and magnetic field signatures of interplanetary coronal mass ejections, Space Sci. Rev., 123, 31-43, 2006. 